\title{
Aging cognition: from neuromodulation to representation
}

\section{Shu-Chen Li, Ulman Lindenberger and Sverker Sikström}

Basic cognitive functions, such as the abilities to activate, represent, maintain, focus and process information, decline with age. A paradigm shift towards cross-level conceptions is needed in order to obtain an integrative understanding of cognitive aging phenomena that cuts across neural, information-processing, and behavioral levels. We review empirical data at these different levels, and computational theories proposed to enable their integration. A theoretical link is highlighted, relating deficient neuromodulation with noisy information processing, which might result in less distinctive cortical representations. These less distinctive representations might be implicated in working memory and attentional functions that underlie the behavioral manifestations of cognitive aging deficits.

Although averagelife expectancy in most societies has increased from about 45 years in 1900 to about 75 years in 1990 (Ref. 1), basic cognitivefunctions decline with advancing age. Thus, the rapid growth of aging populations worldwide is accompanied by an urgency to obtain integrated understanding of mechanisms and processes of cognitive aging at different levels.

Cognitive aging phenomena at different levels Since the first studies on adult age differences in intellectual functioning were published in the 1920s (e.g. Ref. 2), cognitive aging phenomena have been studied at various levels (see Fig. 1). At the behavioral level, individual difference researchers have documented aging-related ded ines in many psychometric measures of fluid intelligence ${ }^{3}$ (i.e. basic cognitive mechanics ${ }^{4}$ for memorizing, reasoning, and

Shu-Chen Li*

Center for Lifespan

Psychology, Max Planck Institute for Human

Development, Lentzeallee 94, D-14195 Berlin,

Germany

*e-mail: shuchen@ mpib-berlin.mpg.de

Ulman Lindenberger School of Psychology, Saarland University, Saarbrücken, Germany.

Sverker Sikström Dept of Psychology, Stockholm University Stockholm, Sweden. learning). F urthermore, aging-related increases of intra-individual variability, inter-individual variability, and de-differentiation of ability structures (increased correlation between different cognitive abilities) are also common observations (see Ref. 5 for review). At theinformation-processing level, experimentally oriented cognitiveaging researchers have proposed general processing resources, such as working memory capacity, attentional mechanisms, and processing speed, as explanatory mechanisms for theage differences in fluid intelligence observed at the behavioral level (seeRefs 6,7 for review). At the neurobiological level, neuroscientists have been studying brain aging at the anatomical ${ }^{8}$, metabolic, and neurochemical levels ${ }^{9}$.
I ntegration across behavioral, informationprocessing, and neurobiological levels has been difficult to establish. Recent advances in neuroimaging and computational neuroscience open new avenues for exploring functional relationships between cognitiveaging phenomena at different levels. The process of integrating data and theories from different levels provideopportunities for related fields toco-evolveby ways of cross-level hypothesis generation and testing ${ }^{10,11}$. In this article, wefirst review empirical data of cognitiveaging at the behavioral, information-processing and neurobiological levels. Wethen consider recent cross-level computational theories ${ }^{5,12-14}$ aiming at integrating findings of aging-related dedines of neuromodulation and various benchmark cognitive aging deficits.

Aging, information processing, and neuromodulation Aging affects three main facets of information processing. People's abilities toactivate, to represent and maintain information in mind, toattend to relevant but ignore irrelevant information, and to process information promptly declinewith advancing age. At the neurobiological level, the efficacy of neuromodulation also declines. Among various neurotransmitter systems, wefocus on the monoamines (e.g. serotonin and the catecholamines, particularly dopamineand noradrenal ine) ${ }^{15-19}$ because they have been studied extensively with respect to ded ines in working memory ${ }^{20}$ and processing speed ${ }^{21}$ during normal aging. Other transmitters al so affect cognitive aging. F or instance, cholinergictransmission is important for long-term memory consolidation ${ }^{22}$, which plays a rolein Alzheimer pathology23, and glutamatesometimes interacts with other transmitters (e.g. dopamine, GABA and acetylcholine) ${ }^{24,25}$.

Deficits in various facets of information processing Working memory function' refers to an ensemble of processes all owing peopleto activate, simultaneously represent and hold information in immediate memory, while operating on thesame or other information. Aging-related ded inein working memory function ${ }^{26}$ has been found in many memory span tasks (e.g. Ref. 27; summary data in Fig. 2a). Besides the more 'traditional' memory capacity view, working memory has recently been decomposed into processes of representing and maintaining context information subserving both mnemonic and attentional control functions ${ }^{12}$.

Aging-related decrements in attentional mechanisms have been found in various selectiveand focused attention tasks (see Ref. 28 for review) and other interferencetasks, such as the Stroop and proactive interferencetasks. Lastly, speed is a ubiquitous aspect of information processing as all processes taketime. Thereis ampleevidencefor agingrelated slowing in many tasks (seeR efs 29,30 for review; summary data adapted from Ref. 27 in Fig. 2b). 


\section{Behavioral level}

What are the age differences in fluid intelligence? Are there aging effects beyond performance level? e.g. aging-related increases in:

- intra-individual performance variance

- inter-individual performance variance

- inter-performance covariations

\section{Information-processing level}

Why are there age differences in fluid intelligence?

Are they related to aging-related declines in processing resources?

e.g. aging-related declines in:

- working memory capacity

- attention regulation/inhibitory function

- processing speed

\section{Neurobiological level}

How are aspects of the aging brain implicated in cognitive aging?

Are aging deficits of information processing related to various

aspects of the aging brain?

e.g. aging-related changes in:

- prefrontal cortex and (or) other neuroanatomical structures

- oxygen/glucose metabolism in different brain regions

- neuromodulation and neuronal noise
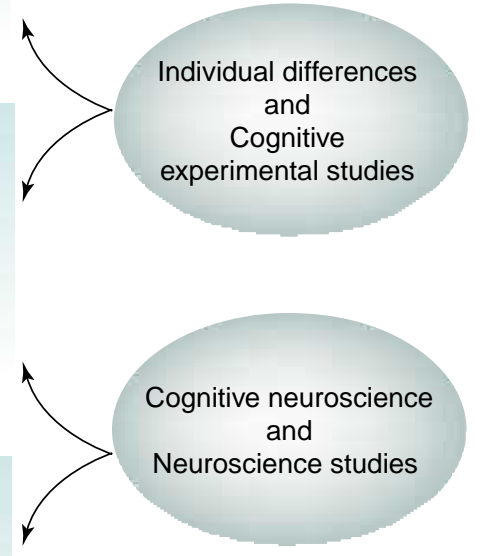

TRENDS in Cognitive Sciences

Fig. 1. A summary of cognitive aging issues addressed by researchers of different specializations working at various levels of analysis.

Limits of resource-reduction theories

Given the above evidence, theories of cognitive aging typically explain behavioral manifestations of cognitive impairments by positing that working memory, attention regulation, and processing speed act as cognitive resources that decline with aging (see Ref. 6 for review). However, two major difficulties confront the resource-reduction theories.

First, although the different processing resources are commonly considered as alternative explanations, they are conceptually and empirically interdependent. For instance, attentional control mechanisms involved in representing and maintaining context information could be important components of working memory ${ }^{12}$. Similarly, speed is an inevitable second-order phenomenon that might reflect the compound effect of all temporal demands incurred from attentional and storage mechanisms involved in processing a given task. Second, the resourcereduction accounts tend to be circular in nature. Reductions in processing resources are assumed to cause cognitive impairments, and, at the sametime, old people's poor performances are taken as indications of resource reductions. It has been suggested that such circularity could be avoided by establishing explicit links between the processing resources and their potential neurobiological underpinnings ${ }^{31}$.
Aging and deficient dopaminergic modulation Severeneuroanatomical degeneration resulting from cell death and reduced synaptic density is typical for pathological aging (e.g. Alzheimer's disease). Recent evidence, however, suggests that milder cognitive deficits occurring during normal aging arelikely to be duemainly to neurochemical shifts in still relatively intact neural circuits ${ }^{32}$. The dopaminergic system is a promising neurochemical correlate of cognitiveaging for several reasons.

First, dopamine transmitter content and binding mechanisms in various brain regions decl ineduring normal aging. Earlier studies focused mostly on dopaminemechanisms in thenigrostriatal region and found a reduction in the number of dopamineD2 receptors of about $10 \%$ per decadestarting at theage of about 20 years ${ }^{15,16}$. Recent findings suggest that dedines in striatal D2 receptors are related to attenuated extrastriatal glucose metabolism ${ }^{18}$. There is also new direct evidence of D2-receptor loss in various extrastriatal regions ${ }^{19}$, such as theanterior cingulate cortex (13\% per decade), frontal cortex (11\% per decade, Fig. 2C), hippocampus (10\%), and theamygdala (7\%). Besides D2 receptors, dopamine D1-receptor loss has also been observed in thestriatum ${ }^{33}$ and frontal cortex ${ }^{34}$, although currently theevidence is not as conclusiveas that for D2-receptor loss. Recently, the rol es of D1 receptors in aging and in schizophrenia have attracted increasing interest. With expanding knowledge of the structureand function of dopamine receptors, the 
Fig. 2. Age-related changes in information processing and neurotransmitter density. (a) Negative adult age differences in working memory measured by three types of span test (computational, reading and backward digit span), scaled in Zscore metric. (b) Negative adult age differences in processing speed measured by three perceptual speed tests (digit symbol substitution, pattern and letter comparison), scaled in a Z-score metric. (a and b adapted with

permission from Ref. 27.) (c) Aging-related declines in dopamine D2-like receptor availability in the frontal cortex. (Adapted with permission from Ref. 19.)

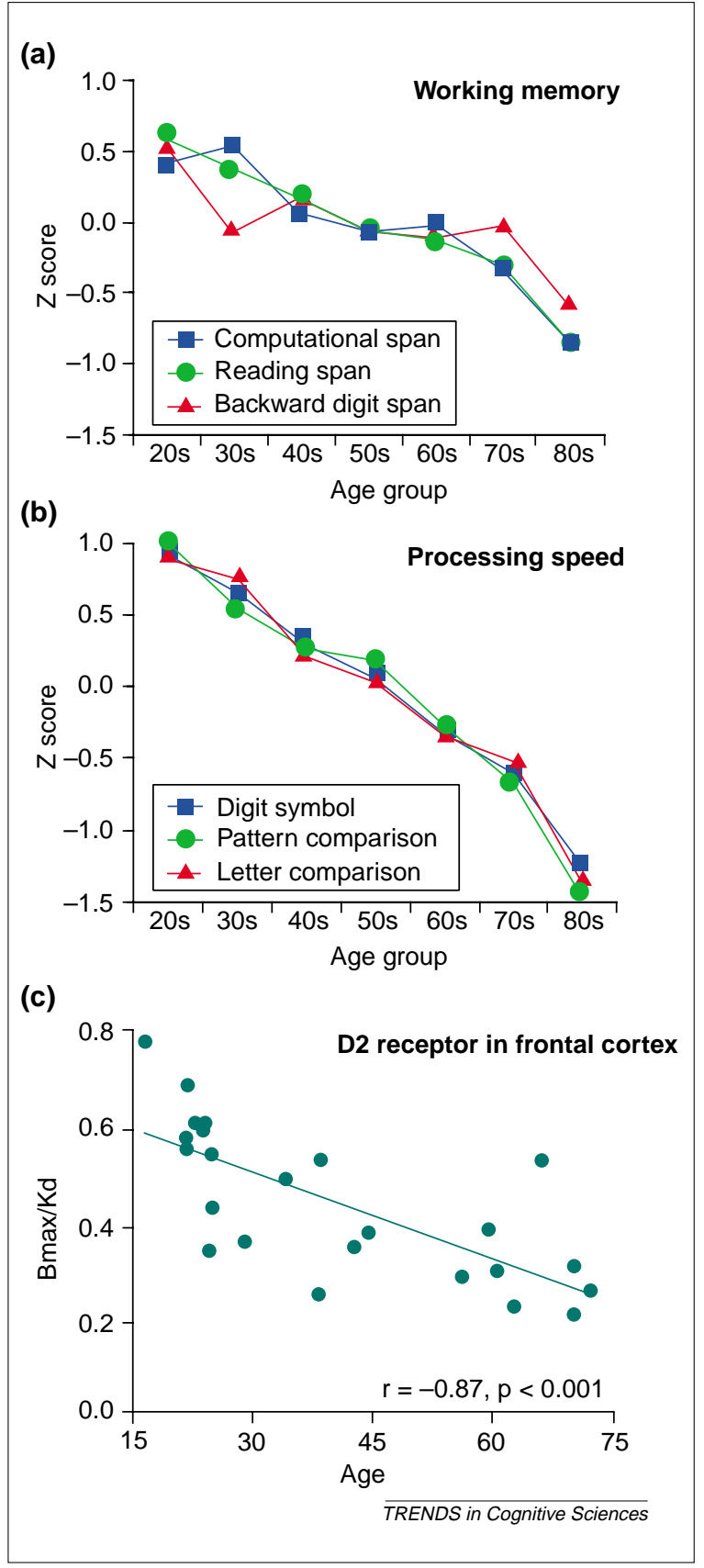

relation between aging and the interactions between D1 and D2 (Refs 35,36), and other receptor subtypes can beinvestigated moresystematically.

Second, cognitive aging deficits have been attributed, at least in part, to prefrontal cortex dysfunction (see Ref. 37 for review). More recent data suggest that the main locus of dysfunction associated with working memory deficits is a morespecific region of thePFC - the dorsal lateral PFC (Ref. 38). Research over the last two decades suggests that dopamine modulates how well thePFC makes use of briefly activated cortical representations to circumvent constant reliance on environmental cues and to regulate attention towards relevant stimuli and appropriate responses ${ }^{20}$. Besides the di rect influence of D2 receptor loss in the PFC, declines in nigrostriatal dopaminemechanisms could also contributetoaging-related PFC dysfunction, as the nigrostriatal area is well interconnected with thePFC via frontal-striatal circuits 39,40 .

Third, besides the apparent parallelism between aging-related ded ines in working memory, processing speed, and D2 receptors across theadult lifespan (Fig. 2), thereis also more direct experimental evidence for functional relationships between deficient dopaminergic modulation and cognitiveded ines. For instances, reduced dopamine receptor density in old rats'nigrostriatum is associated with decreased response speed and increased reaction time variability ${ }^{21}$. Drugs that facilitate dopaminergic modulation (e.g. D1 agonists) al leviate working memory deficits of aged monkeys with naturally occurring dopaminedepletion in their PFC (R ef. 41). In humans, aging-related attenuation of the striatal D2-receptor binding mechanism is statistically associated both with decreased glucose metabolism in extrastriatal cortical regions innervated by dopaminergic pathways ${ }^{18}$ and with age differences in processing speed and epi sodic memory ${ }^{42}$.

Taken together, deficient dopaminergic modulation is implicated in cognitiveaging deficits; however, the details of this link between neuromodulation and cognition await further explication. At the cellular level, empirical and theoretical investigations aimed at understanding how dopaminergic modulation affects the memory field and signal integration of PF C neurons haverecently begun ${ }^{43-46}$. At a more molar level, studies areal so underway that are exploring computational principles that might relate dedines in neuromodulation to cognitive aging deficits observed at theinformation-processing and behavioral levels ${ }^{12,13}$.

\section{Recent computational theories linking neuromodulation with cognitive aging}

In 1990, two mathematical theories of cognitiveaging were proposed in part to resolvetheinterdependence and circularity problems facing the resource-reduction theories ${ }^{47,48}$. Although not operating at thelevel of neuromodulation, both theories foreshadowed crosslevel conceptual orientation. Thenetwork-disconnection theory of aging and information-processing rate ${ }^{47}$ makes broad reference to neuroanatomical changes that might invol vethe degeneration of axonal connections. Theinformation-loss theory of agingrelated cognitiveslowing ${ }^{48}$ assumes an increasing rate of neural information loss across processing steps.

Although both theories are oriented towards linking behavioral cognitiveaging phenomena with conceivable properties of the aging brain, they do not suggest explicit neuronal mechanisms for attenuated axonal connections or increased information loss. Neuromodulation of synaptic transmission is a natural starting point for further theorizing about these missing links. I $n$ thelight of accumulating evidencefor aging-related deficiency of dopaminergic 


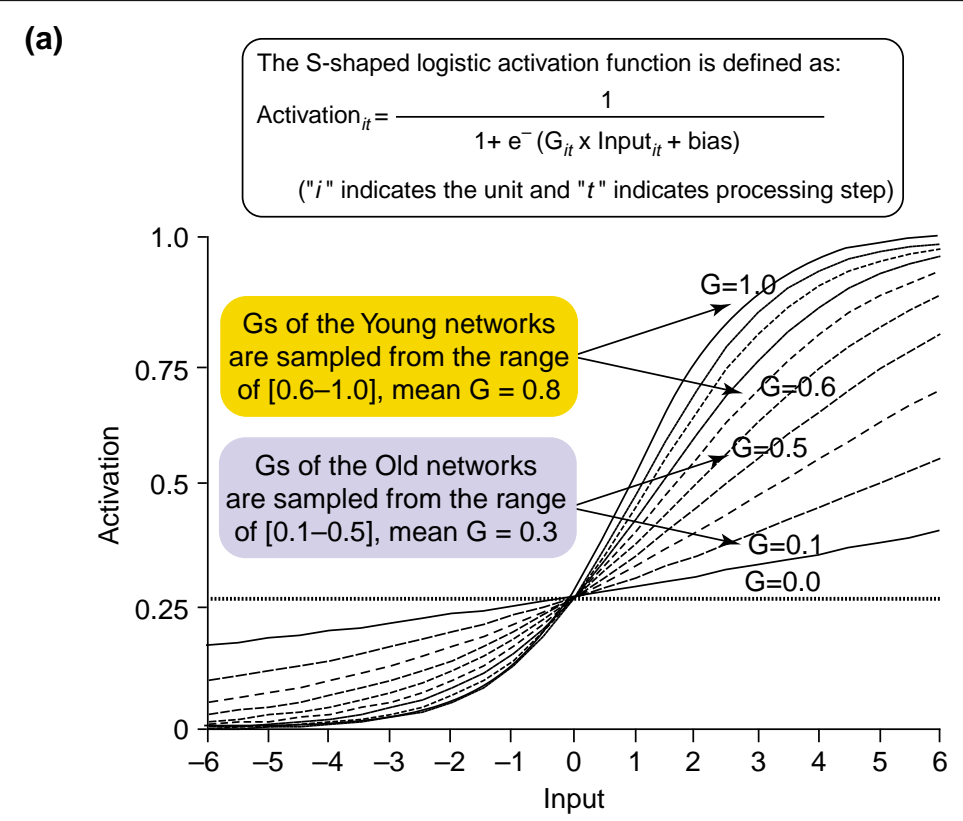

(b)

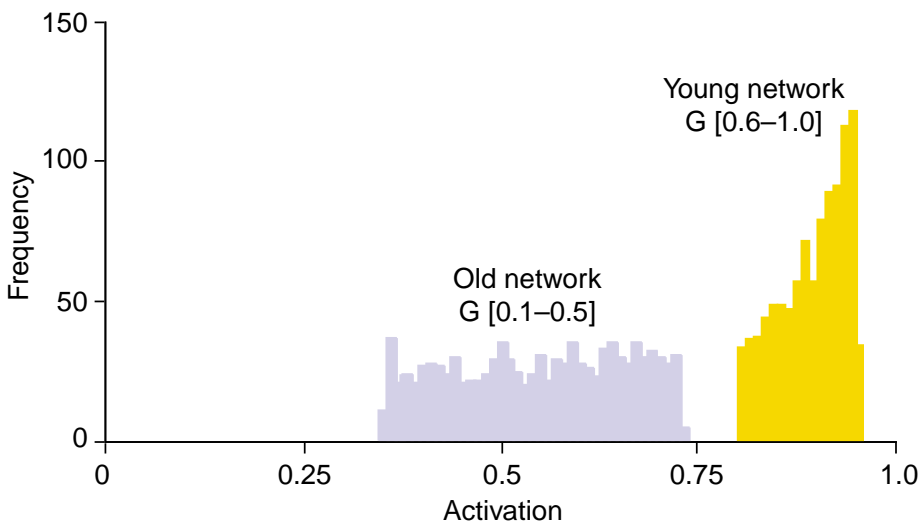

(c)

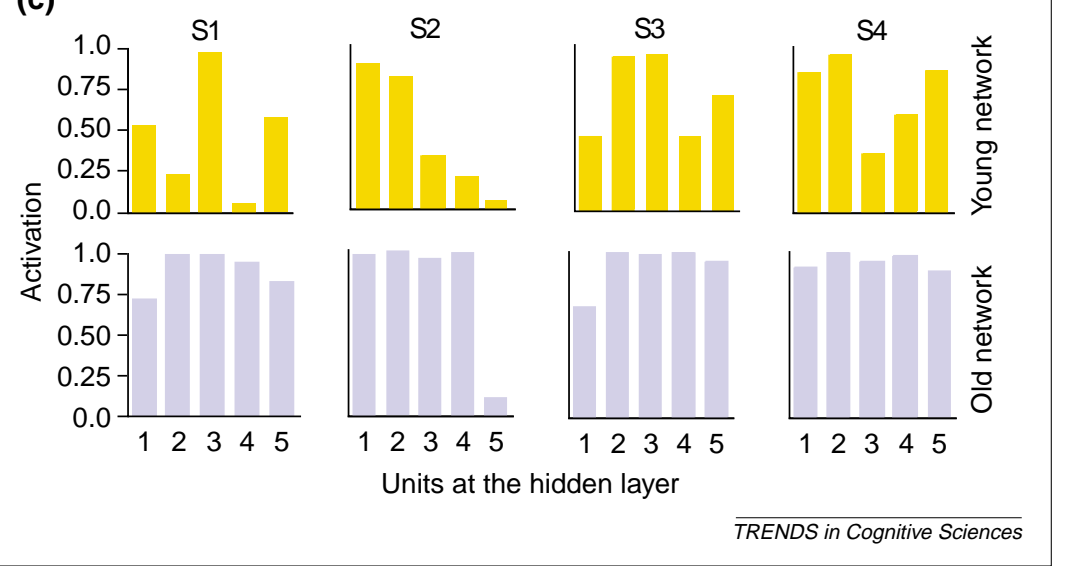

Fig. 3. Simulations from computational theories of cognitive aging: effects of deficient neuromodulation. (a) The S-shaped logistic activation function at different values of the gain parameter, G. Physiological evidence suggests that the logistic function with a negative bias captures the function relating the strength of an input signal to a neuron's firing rate, with its steepest slope around the baseline firing rate. Reducing mean $\mathrm{G}$ flattens the activation function such that a unit becomes less responsive. Aging-related decline of dopaminergic modulation can be simulated by sampling values of $\mathrm{G}$ from a distribution with a lower mean. (b) $G$ and the variability of activation across processing steps. Reducing mean $\mathrm{G}$ ( 0.8 and 0.3 for the 'young' and 'old' networks, respectively) increases the temporal variability of a unit's response to an identical input signal (set to 4.0) across 1000 trials. (c) Internal activation patterns across five hidden units of one 'young' and one 'old' network in response to four different stimuli (S1 to S4). The internal representations of the four stimuli are much less differentiable in the 'old' than in the 'young' network. (Adapted from Ref. 13 with permission.) modulation, more recent computational inquiries ${ }^{12,13}$ explore mechanisms that could explicateaspects of the functional relationships between aging-related dedinein dopaminergic modulation, neural information processing fidel ity, and cognitiveaging.

Although dopamine's modulatory effects vary widely, depending on cortical region and receptor type, a general feature of dopaminergicmodulation can be conceptual ized as altering thesignal-to-noise ratio of neural information processing, thus regulating neurons' sensitivity to afferent signals. Oneway to model this effect is adjusting thegain (G) parameter of the sigmoidal activation function in feedforward backpropagation networks ${ }^{49}$. Other approaches, focusing specifically on modeling voltage-dependent dopaminergicmodulation of PFC neurons' memory fields in recurrent networks, havealso been proposed 44,45 . Although differing in implementation details, theoverall neuromodulatory effect of tuning the signal-to-noiseratiois a common feature shared by these approaches.

Recently, two complementary computational theories extended the approach of manipulating the $G$ parameter of thesigmoidal activation function ${ }^{49}$ to model aging-related attenuation of dopaminergic modulation and cognitive aging deficits. Onetheory focuses on capturing functional interactions between dopaminergicmodulation and the dorsal lateral PFC in regulating context representation and maintenance ${ }^{12}$. Operating at thelevel of neural signal processing in general, theother theory aims at elucidating a potential sequence of functional relations from deficient dopaminergic modulation to reduced neural information processing fidelity with ensuing consequences for cortical representational distinctiveness and cognitive aging deficits ${ }^{13}$.

\section{From deficient neuromodulation to neural noise}

A dassical hypothesis of cognitiveaging at the neurobiological level is increased neural noise (haphazard activation during neuronal information processing $)^{50}$. However, thus far, mechanisms leading to such an increaseand its proximal and distal consequences havenot been unveiled. Simulating aging-related dedine of dopaminergic neuromodulation by attenuating the $G$ parameter in neural networks hints at a possiblechain of mechanisms relating deficient neuromodulation to increased neural noise and less distinctive cortical representations.

\section{Reduced responsivity and increased noise in neural information processing}

Conceptually, the G parameter captures dopaminergicmodulation by altering the slope of the activation function. Reducing G simulates agingrelated attenuation of dopaminergic modulation by reducing thesl opeand flattening the non-linearity of the S-shaped logisticactivation function, such that a unit's average responsivity to excitatory and inhibitory input signals is reduced (Fig. 3a). 
Furthermore, if the values of a unit's $G$ across processing steps are randomly chosen (i.e. stochastic G; Ref. 13) from a set of values with a lower average, the unit's responseto a given external signal becomes more variable, which implies a decrease in signal transmission fidelity (see Fig. 3b). Put differently, a given amount of random variations in $\mathrm{G}$, simulating random fluctuations of transmitter release ${ }^{51}$, generates more haphazard activation during signal processing if theaverage value of $\mathrm{G}$ of the processing units is reduced. This sequence of effects computationally depicts a potential neurochemical mechanism for aging-related increasein neural noise: as aging attenuates neuromodulation, the impact of transmitter fluctuations on the overall level of haphazard neuronal activity is amplified in theaging brain.

\section{Less distinctive cortical representations}

Thecomputational simulations further show that as reduced responsivity leads to increased intra-network random activation variability, another subsequent effect is a decrease in the distinctiveness of the network's internal representations. Low representational distinctiveness means that the activation profiles formed across the network's hidden units for different stimuli are less readily differentiable from each other. Toillustrate this, Fig. 3c shows the internal activation patterns captured by the activity levels across units of the hidden layer of a 'young' (higher average G) and an 'old' (lower average G) network in responseto four input signals. As can be seen, theinternal stimulus representations are less distinctivein the 'old'than in the 'young' network.

In terms of everyday examples, this effect implies that, as peopleage, mental representations of various events and the contexts within which the events occurred, such as conversations held with different individuals within a day in different social settings, becomeless distinct, and thus aremore confusable with each other. This set of simulation results provides a computational analog for an earlier information-processing hypothesis, which suggests that old people's memory traces for encoded events areless distinctive because ol d peopleprocess information less elaborately than young people as a result of reduced attentional resources ${ }^{52}$. Couched within the cross-level theoretical framework, the simulation also suggests that deficient dopaminergic modulation of the PFC's attention regulation mechanisms might bethe neural correlate of less elaborateprocessing.

Furthermore, wehave recently shown that such computational effects (i.e. reduced representational distinctiveness as a result of lowering stochastic $G$ ) also generalize to networks with multiple processing modules (S-C. Li and S. Sikström, unpubl ished data). Reducing the mean $\mathrm{G}$ of units within two distinct processing modules leads to extensiveactivation overlap across modules.
Taken together, a potential biological implication of thesetheoretical effects could bethat as dedining dopaminergic modulation drives down cortical neurons'responsivity and increases neural noisein the aging brain, cortical representations elicited by different stimuli and contexts become less differentiated as peopleage. Cortical representations of concurrent external events (perception) and later reinstatements of theseevents (memory) arethe primitives of subsequent cognitive processing carried out by various neural circuits. Therefore, deficient neuromodulation causingless distinctive cortical representations of different events and contexts may havefar-reaching consequences for various facets of cognition. Thetheoretical link laid out herewas tested and supported by a series of simulations capturing behavioral human cognitiveaging phenomena (see Box 1). In this article, wehave focused on dopaminergic modulation because of the converging evidence with respect to its functional effects on working memory, attention and processing speed, along with ded ines in its content and receptor mechanisms in various brain regions during normal aging. However, the computational formalisms demonstrated here could begeneral ized to other transmitter systems if they exhibit similar functional properties and aging gradients.

\section{Implications: a paradigm shift towards co-evolving fields across levels}

Details regarding theinvolvement of neuromodulation in cognitive aging deficits remain to be unraveled. Pieces of the puzzle are emerging in various sub-fields, and the field as a wholecould benefit from a paradigm shift towards overarching frameworks seeking to integrate cognitiveaging phenomena across different levels. The proposed theoretical link - from attenuated neuromodulation to increased neural noiseand less distinctivecortical representations in the aging brain, and finally on to cognitiveaging deficits - is only an initial proposal awaiting further vigorous empirical testing. Nevertheless, neural computational theories of the kind reviewed here ${ }^{12,13}$ integrate evidence of agingrelated ded ine of dopaminergic modulation with a broad range of human cognitiveaging phenomena and suggest expl icit mechanisms that could giverise to the functional relations - a task unlikely to be accomplished by either animal neurobiological or human neuroimaging studies al one.

A shift to cross-level paradigms generates more opportunities for hypothesis generation and testing across levels. For instance, neuromodulation might not only influenceaging-related increases in intraindividual performancevariability within individuals ${ }^{53,54}$, but also inter-individual diversity at thegroup level. Futureanimal pharmacological studies could directly examinethe effects of dopamineagonists and antagonists on intra-individual fluctuations and their effects on inter-individual diversity. 


\section{Questions for future research}

- Is more distinctive stimulus representation computationally equivalent to sparse memory representation? How might spare memory representation be formally related to more efficientmemory capacity and processing speed?

- Can differences in behavioral manifestations between cognitive aging and schizophrenic syndrome be linked to differences in the relative degree of impairment in various subtypes of dopamine receptors?

- What role does neuromodulation, in general, and the dopaminergic system, in particular, play in the course of normal cognitive development in childhood and in developmental disorders of attention? To what extent can child cognitive development be conceived of as an increase in the efficacy of neuromodulation and cortical representations?

Recent neuroimaging evidencesuggests that cognitive processes that arecarried out separately by either theleft or right hemisphere in young adults coactivate both hemispheres in ol d people. F or instance, peoplein their 60 s and beyond showed bilateral activity when retrieving items from memory ${ }^{55}$ or performing verbal and spatial working memory tasks ${ }^{56}$. Currently, these data are primarily interpreted in terms of a compensation view: suggesting that the increased bilateral activation in old adults might be one way to compensatefor neurocognitive deficits ${ }^{57}$. There is some supporting evidence for this view.

For instance, memory performance of old adults who exhibit bilateral activity is better than that of those who do not ${ }^{56}$ (Cabeza, Anderson, Kester, and Rajah, unpublished data). The recent finding of an association between striatal D2 receptor availability and gl ucose metabol ism in thefrontal cortex ${ }^{18}$ raises thequestion of whether deficient neuromodulation and theincrease in bilateral activation might berelated.

Aging-related ded ines in neuromodulation could be one aspect of neurocognitive deficits needing compensation.

Acknowledgements

The authors are grateful to Valtteri Kassinen and Denise C. Parkfor permitting the redrawing of some data from their studies. We thank Todd S. Braver for helpful e-mail exchanges about the relations between his theory and ours. We are grateful to Naftali Raz, the anonymous reviewers, and J ulia Delius for their many helpful comments on an earlier version of this article. We thank Peter A. Frensch for previous contributions to related work, and Paul B. Baltes and the Max Planck Institute for sponsoring this project. S-C.L. thanks Stephen Lewandowsky for pointing out issues about gain regulation and interference in neural networks during the supervision of her $\mathrm{PhD}$ dissertation in 1993. The effect of attenuating the $G$ parameter, thus causing less distinctiveinternal representations and increased overlapping activation in different informationprocessing pathways (S-C. Li and S. Sikström, unpubl ished data), suggests that deficient bilateral activation might partly berelated to deficient neuromodulation, in addition to reflecting possible compensatory reorganization of functional brain circuitry or compensatory behavioral strategies.

This review has focused on relating the different levels of cognitiveaging phenomena, tracing a link from neuromodulation to cognition to behavior. I $n$ the foreseeablefuture, however, it will benecessary to examinemore actively reciprocal influences from behavior to cognition to neural mechanisms, and to use the knowledge gained from basic research in reallife applications. This includes, for example, research on behavioral training that assists older adults in developing compensatory cognitive strategies (e.g. the use of mnemonics, external memory cues, and other environmental and contextual support) ${ }^{58}$ that capitalizeon using the cortical plasticity that the aging brain seems still to possess ${ }^{59}$.
Box 1 Simulations linking neuromodulation with behavioral data

Leaming rate, asymptotic performance, and interference susceptibility

With advancing age people take longer to learn paired associates (arbitrary word pairs, such as 'computer-violin'). In agreement with empirical findings that compared people in their 20 s with those in their 50s (Ref. a), simulations show a comparable drop in performance: the 'old' networks (i.e. having a reduced mean gain, $\mathrm{G}$ ) require more trials than the 'young' networks to reach increasingly strict recall criteria in pairedassociate learning (Fig. la).

Besides slower learning rate, ample data about the effects of aging and practice on skill acquisition show that aging-related decrements persist even at old people's asymptotic performance levels $\mathrm{s}^{\mathrm{b}}$, a phenomenon that can also be accounted for by reducing the average $\mathrm{G}$ of the network's processing units (Fig. Ib).

Another prominent cognitive aging deficit is older people's increasing susceptibility to interference. In the context of paired-associate learning, sixty-year-olds are more susceptible than forty-year-olds to interference of previously learned word pairs with the learning of new pairs, and they need more trials to learn new word pairs if interference is strong ${ }^{c}$. In line with the empirical evidence, the number of trials required for learning new word pairs under conditions of weak and strong interference differ more in the 'old' than in the 'young' networks (Fig. Ic).

\section{Performance variability and covariation}

Behavioral data also show aging-related increases of performance variations within a single individual across time (or different tasks), and differences across individuals, as well as covariation between tasks ${ }^{d}$. Aging effects on variance and covariation can also be accounted for by mean $\mathrm{G}$ reduction, suggesting that aging-related increase of intraindividual performance variability, inter-individual diversity, and ability de-differentiation might all be associated with decreasing efficacy of neuromodulation.

Simulations in which intra-network variability was tested by measuring a given network's performance variability across study lists in four conditions of paired-associate learning, show that the magnitude of average intra-network performance variability was larger in the 'old' than in the 'young' networks, across all conditions (Fig. Id). Across long and short lists, inter-network performance variability was also larger among the old networks (Fig. le). Furthermore, correlations between the performances across conditions were higher in the group of 'old' than in the group of 'young' networks (Fig. If). 
(a)

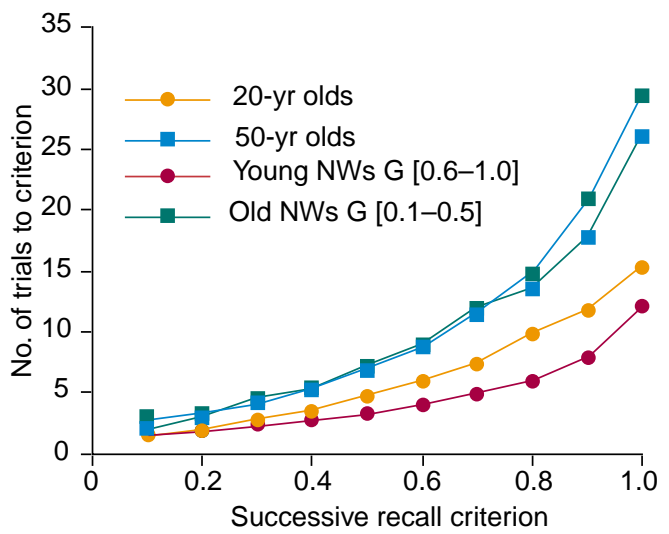

(c)

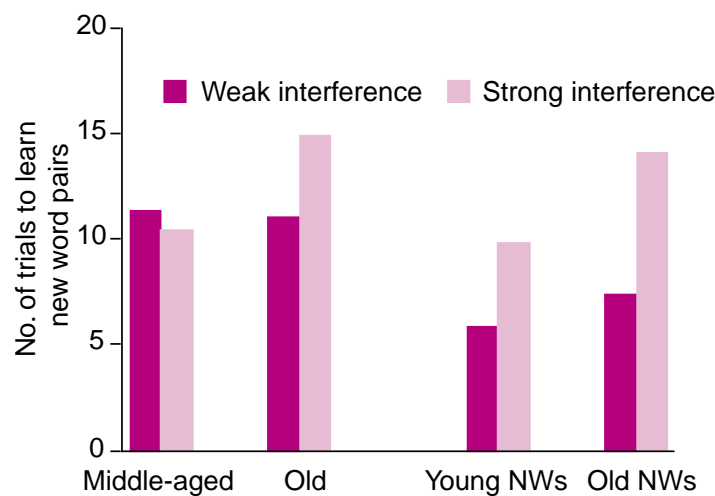

(e)

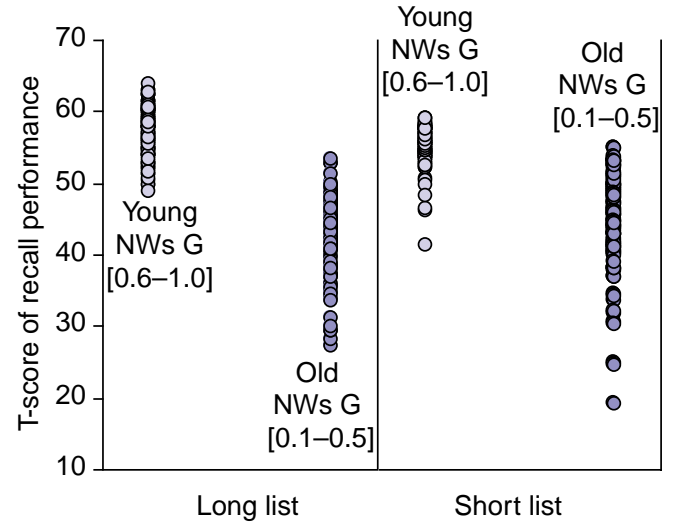

(b)

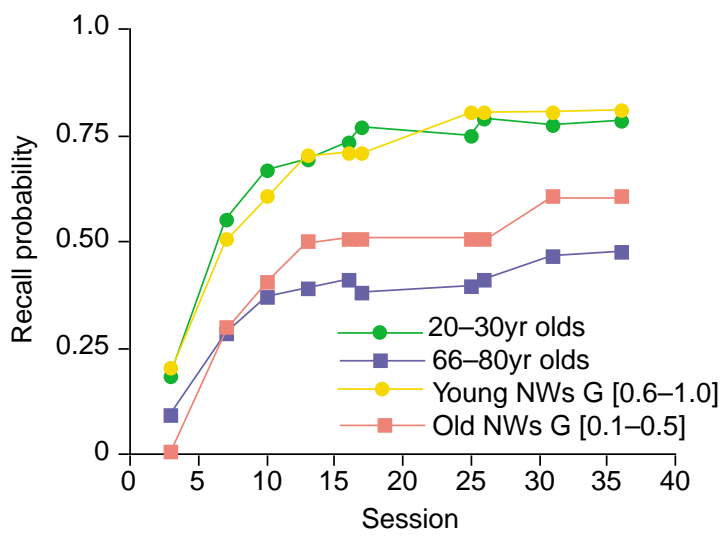

(d)

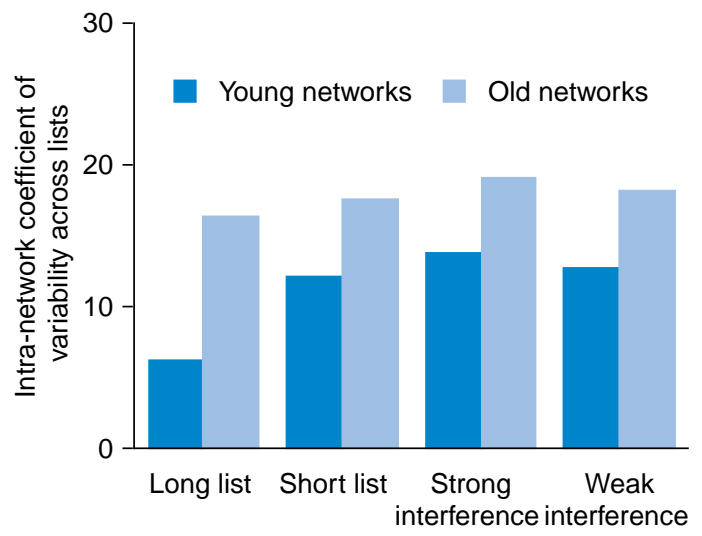

(f)

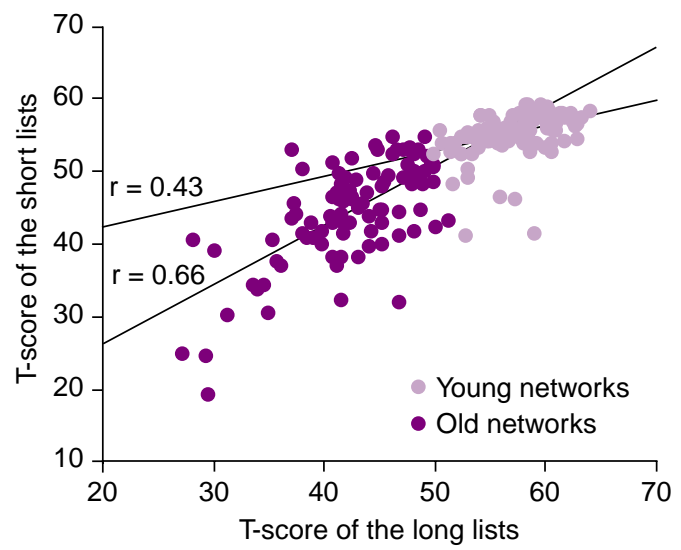

$\overline{T R E N D S}$ in Cognitive Sciences

\section{References}

a Monge, H.R. (1971) Studies of verbal learning from the collegeyears through middleage. J. Gerontol . 26, 324-329

b Baltes, P.B. and Kliegl, R. (1992) Further testing of thelimits of cognitive plasticity: negativeage differences in a mnemonic skill are robust. Dev. Psychol. 28, 121-125

c Lair, C.V. et al. (1969) Associativeinterference in the paired-associatelearning of middle-aged and old subjects. Dev. Psychol. 5, 548-552

d Lindenberger, U. and Baltes, P.B. (1997)

Intellectual functioning in old and very old age: cross-sectional results from theBerlin Aging Study. Psychol. Aging 12, 410-432
Fig. I. Comparing simulations with human behavioral data. (a) Aging deficits in paired-associate learning in human subjects and simulations. There is good agreement between the simulations and human data: like the 50-yr olds the 'old' networks (NW) required more trials to reach harder recall criteria. (b) Aging impairments at asymptotic performance in human subjects and simulations. The human performance is reasonably well simulated by reducing the average gain $(G)$ of the network's processing units. (c) Increases in susceptibility to interference in dual-list paired-associate learning are seen both in human subjects and in young and old network simulations. (d) The effect of mean $\mathrm{G}$ reduction on intra-network variability in performance level across different study lists in four conditions. The old networks (lower mean $G$ ) show a greater intra-network variability. (e) The G parameter and inter-network variability. Across different list lengths, reducing mean $\mathrm{G}$ not only reduces mean recall performance, but also increases inter-network variability. (f) $G$ and covariation of performances. Reducing mean $G$ increases the correlation between performances with short and long lists. The correlation is stronger for the 'old' $(r=0.66)$ than for the 'young' $(r=0.43)$ networks (difference between correlations is statistically significant, $z=2.4$ ). (Adapted with permission from Ref. 13.) 


\section{References}

1 Kannisto, V. (1994) Devel opment of Ol dest-old Mortality, 1950-1990: Evidencefrom 28 Developed Countries, OdenseUniversity Press

2 Foster, J.C. and Taylor, G.A. (1920) The applicability of mental tests to persons over 50 . J . Appl. Psychol . 4, 39-58

3 Horn, J .L. (1982) Thetheory of fluid and crystallized intelligencein relation to concepts of aging in adulthood. In Aging and Cognitive Processes (Craik, F.I.M. and Trehub, S., eds), pp. 237-278, Plenum Press

4 Baltes, P.B. (1987) Theoretical propositions of lifespan developmental psychology: on the dynamics between growth and decline. Dev. Psychol . 23, 611-626

5 Li, S-C. and Lindenberger, U. (1999) Cross-level unification: a computational exploration of thelink between deterioration of neurotransmitter systems and dedifferentiation of cognitiveabilities in old age. In Cognitive Neuroscience of Memory (Nilsson, L-G. and Markowitsch, H., eds), pp. 103-146, Hogrefe and Huber

6 Salthouse, T.A. (1991) Theoretical Perspectives on CognitiveAging, Erlbaum

7 Craik, F.I.M. and Salthouse, T.A., eds (2000) The Handbook of Aging and Cognition, Erlbaum

8 Raz, N. (2000) Aging of the brain and its impact on cognitive performance: integration of structural and functional findings. In The Handbook of Aging and Cognition (Craik, F.I.M. and Salthouse, T.A., eds), pp. 1-90, Erlbaum

9 Schneider, E.L. et al. (eds.), (1996) Handbook of theBiology of Aging, Academic Press

10 Churchland, P.S. and Sejnowski, T.J . (1988) Perspectives on cognitiveneuroscience. Science 242, 741-745

11 Schacter, D.L. (1992) Understanding implicit memory. Am. Psychol . 47, 559-569

12 Braver, T.S. et al. Context processing in older adults: evidence for a theory relating cognitive control to neurobiology in healthy aging. J . Exp. Psychol. Gen. (in press)

$13 \mathrm{Li}, \mathrm{S}-\mathrm{C}$. et al. (2000) Unifying cognitiveaging: from neuromodulation to representation to cognition. Neurocomputing, 32-33, 879-890

$14 \mathrm{Li}, \mathrm{S}-\mathrm{C}$. Connecting the many levels and facets of cognitiveaging. Curr. Dir. Psychol. Sci. (in press)

15 Wong, D.F. et al . (1984) Effects of age on dopamine and serotonin receptors measured by positron tomography in theliving human brain. Science, 226, 1393-1396

16 Wong, D.F. et al . (1997) Quantification of neuroreceptors in theliving human brain: III. D2-likedopamine receptors: theory, validation and changes during normal aging. J . Cereb. Blood Flow Metab. 17, 316-330

17 Arnsten, A.F.T. (1999) Age-related cognitive deficits and neurotransmitters: the role of catecholaminemechanisms in prefrontal cortical cognitivedecline. In Neurodegenerativeand Age-Related Changes in Structureand F unction of Cerebral Cortex (Cerebral Cortex Vol. 14) (Peters, A. and Morrison, J ., eds), pp. 89-110, Plenum Press

18 Volkow, N.D. et al. (2000) Association between age-related decline in brain dopamineactivity and impairment in frontal and cingulate metabolism. Am. J . Psychiat. 157, 75-80
19 Kaasinen, V. etal. (2000) Age-related dopamine D2/D3 receptor loss in extrastriatal regions of the human brain. Neurobiol. Aging 21, 683-688

20 Arnsten, A.F.T. (1998) Catecholaminemodulation of prefrontal cortical cognitivefunction. Trends Cognit. Sci. 2, 436-447

21 MacRae, P.G. etal. (1988) Reaction time and nigrostraital dopaminefunction: the effects of ageand practice. Brain Res. 451, 139-146

22 Hasselmo, M.E. (1999) Neuromodulation: acetylcholineand memory consol idation. Trends Cognit. Sci. 3, 351-359

23 Bartus, R.T. (2000) On neurodegenerative diseases, models, and treatment strategies: lessons learned and lessons forgotten a generation foll owing the chol inergic hypothesis. Exp. Neurobiol. 163, 495-529

24 Segovia, G. etal. (2001) Glutamatergic neurotransmission in aging: a critical perspective. Mech. Ageing Dev. 122, 1-29

25 Grachev, I.D. et al. (2001) Aging alters the multichemical networking profile of the human brain: an in vivoH-1-MRS study of young versus middle-aged subjects. J . Neurochem. 77, 292-303

26 Grady, C.L. and Craik, F.I.M. (2000) Changes in memory processing with age. Curr. Opin. Neurobiol. 10, 224-231

27 Park, D.C. et al . (1996) Mediators of long-term memory performanceacross thelifespan. Psychol. Aging 4, 621-637

28 McDowd, J .M. and Shaw, R.J . (2000)Attention and aging: a functional perspective. In The Handbook of Aging and Cognition (Craik, F.I.M. and Salthouse, T.A., eds), pp. 221-292, Erlbaum

29 Cerella, J . (1985) I nformation processing rates in the el derly. Psychol. Bull. 98, 67-83

30 Salthouse, T.A. (1996) The processing-speed theory of adult age differences in cognition. Psychol. Rev. 103, 403-428

31 Salthouse, T.A. (1988) Resource-reduction interpretations of cognitive aging. Dev. Rev. 8, 238-272

32 Morrison, J .H. and Hof, P.R. (1997) Lifeand death of neurons in theaging brain. Science 278 , $412-429$

33 Giorgi, O. et al. (1987) D1 dopaminereceptors labeled with ${ }^{3} \mathrm{H}-\mathrm{SCH}$ 23390: decreasein the striatum of aged rats. Neurobiol . Aging 8, 51-54

34 deKuyser, J . et al . (1990) The effect of aging on the D1 dopaminereceptors in human frontal cortex. Brain Res. 528, 308-310

35 Morgan, D.G. et al. (1987) Divergent changes in D1 and D2 dopamine binding sites in human brain during aging. Neurobiol. Aging 8, 195-201

36 Murray, A.M. and Waddington, J .L. (1991) Agerelated changes in the regulation of behavior by D1:D2 dopamine receptor interactions. Neurobiol . Aging 12, 431-435

37 West, R.L. (1996) An application of prefrontal cortex function theory to cognitiveaging. Psychol Bull. 120, 272-292

38 Rypma, B. and D'Espositio, M. (2000) I solating the neural mechanisms of age-related changes in human working memory. Nat. Neurosci. 2, 509-515

39 Graybiel, A.M. (1990) Neurotransmitters and neuromodulators in the basal ganglia. Trends Neurosci. 13, 244-254

40 Rubin, D.C. (1999) Frontal-striatal circuits in cognitiveaging: evidence for audate involvement. Aging Neuropsychol. Cognit. 6, 241-259
41 Arnsten, A.F.T. et al. (1994) DopamineD1 receptor mechanisms in the cognitive performance of young adult and aged monkeys. Psychopharmacology 116, 143-151

42 Bäckman, L. et al. (2000) Age-related cognitive deficits mediated by changes in the striatal dopamine system. Am. J . Psychiat. 157, 635-637

43 Goldman-Rakic, P.S. et al. (2000) D1 Receptors in prefrontal cells and circuits. Brain Res. Rev. 31 295-301

44 Camperi, M. and Wang, X.J . (1998)A model of visuospatial working memory in prefrontal cortex: recurrent network and cellular bistability. J . Comput. Neurosci. 5, 383-405

45 Lisman, J .E. et al. (1998) A rolefor NMDAreceptor channels in working memory. Nat. Neurosci. 1, 273-275

46 Seamans, J.K. et al. (2001) Bidirectional dopaminemodulation of GABAergic inhibition in prefrontal cortical pyramidal neurons. J. Neurosci. 21, 3628-3638

47 Cerella, J . (1990) Aging and informationprocessing rate. In Handbook of thePsychology of Aging (BirrenmJ .E. and Schaie, K.W., eds), pp. 201-221, Academic Press

48 Myerson, J . et al. (1990) Theinformation-loss model: a mathematical theory of age-related cognitiveslowing. Psychol. Rev. 97, 475-487

49 Servan-Schreiber, D. et al. (1990) A network model of catecholamine effects: gain, signal-tonoise ration, and behavior. Science249, 892-895

50 Welford, A.T. (1965) Performance, biological mechanisms and age: a theor etical sketch. In Behavior, Aging, and theNervous System (Welford, A.T. and Birren, J .E., eds), pp. 3-20, Thomas

51 Hessler, N.A. et al. (1993) The probability of transmitter release at a mammalian central synapse. Nature 366, 569-572

52 Craik, F.I.M. (1983) On thetransfer of information from temporary to permanent memory. Philos. Trans. R. Soc. London Ser. B. 302, 341-359

53 Hultsch, D.F. et al. (2000) Intra-individual variability in cognitive performancein older adults: comparison of adults with mild dementia, adults with arthritis, and healthy adults. Neuropsychol. 14, 588-598

54 Li, S-C. et al. (2001) Short-term fluctuations in el derly people's sensorimotor functioning predict text and spatial memory performance(The MacArthur Successful Aging Studies). Gerontology 47, 100-116

55 Cabeza, R. et al. (1997)Agerelated differences in effectiveneural connectivity. NeuroReport 8, 3479-3483

56 Reuter-Lorenz, P.A. et al. (2000) Age differences in the frontal lateralization of verbal and spatial working memory revealed by PET. J . Cogn. Neurosci. 12, 174-187

57 Cabeza, R. Hemispheric asymmetry reduction in older adults: the Harold model. Psychol. Aging (in press)

58 Li, K.Z.H. etal. (2001) Walking whilememorizing: a SOC study of age-related differences in compensatory behavior under dual-task conditions. Psychol. Sci. 12, 230-237

59 Gross, C.G. (2000) Neurogenesis in the adult brain: death of a dogma. Nat. Rev. Neurosci. 1, 67-73 